\title{
Prevalence of Hymenoptera venom allergy and sensitization in the population-representative German KORA cohort
}

\author{
Simon Blank · Stephanie Haemmerle · Teresa Jaeger • Dennis Russkamp · Johannes Ring • Carsten B. Schmidt- \\ Weber · Markus Ollert
}

Received: 29 October 2018 / Accepted: 11 December 2018 / Published online: 9 January 2019

(C) The Author(s) 2019

\begin{abstract}
Purpose Allergic reactions to Hymenoptera venoms represent potentially life-threatening conditions. However, studies on their prevalence in Germany and their relation to specific IgE sensitization are rare. The aim of this study was to evaluate the prevalence of Hymenoptera venom allergy as well as the frequency of venom-specific IgE sensitization in a large population-based adult German cohort.

Methods Questionnaire data were collected from the participants of the German population-based KORA
\end{abstract}

S. Blank and S. Haemmerle contributed equally to this work.

Electronic supplementary material The online version of this article (https://doi.org/10.1007/s40629-018-0089-4) contains supplementary material, which is available to authorized users.

PD Dr. S. Blank $(\bowtie) \cdot$ D. Russkamp · C. B. Schmidt-Weber Center of Allergy and Environment (ZAUM), Technical University of Munich and Helmholtz Center Munich, Member of the German Center of Lung Research (DZL), Ingolstädter Landstraße 1, 85764 Munich, Germany simon.blank@tum.de

S. Haemmerle · T. Jaeger · J. Ring · Prof. Dr. M. Ollert Department of Dermatology and Allergy Biederstein, Technical University of Munich, Munich, Germany

\section{S. Haemmerle}

Department of Child and Adolescent Psychiatry,

Psychosomatics and Psychotherapy, University Hospital Munich, Munich, Germany

Prof. Dr. M. Ollert $(\bowtie)$

Department of Infection and Immunity, Luxembourg

Institute of Health (LIH), 29, rue Henri

Koch, 4354 Esch-sur-Alzette, Luxembourg

markus.ollert@lih.lu

Department of Dermatology and Allergy Center, Odense Research Center for Anaphylaxis, University of Southern Denmark, Odense, Denmark
(Cooperative Health Research in the Region of Augsburg) S4 baseline study population $(n=4261)$ and the follow-up F4 study population $(n=3074)$, which was conducted seven years later. Moreover, sIgE antibodies to honeybee (HBV) and yellow jacket venom (HJV) as well as to common aeroallergens were measured in the S4 study population.

Results The prevalence of systemic sting reactions ranged between $2.3 \%$ and $2.6 \%$. sIgE sensitization $\left(\geq 0.35 \mathrm{kU}_{\mathrm{A}} / \mathrm{L}\right)$ to $\mathrm{HBV}$ and $\mathrm{YJV}$ was demonstrated in $23.1 \%$ and $31.7 \%$ of the population, respectively (41.6\% to HBV and/or YJV). Double-sensitization to both venoms occurred in $13.2 \%$ of the individuals. Approximately $53 \%$ and $77 \%$ of the individuals who reported shock symptoms after honeybee and yellow jacket stings, respectively, exhibited $\operatorname{sigE} \geq 0.35 \mathrm{kU}_{\mathrm{A}} / \mathrm{L}$ to the culprit venom. In contrast, only $2.8 \%$ of the venom-sensitized individuals reported symptoms exceeding local reactions. Local reactions were reported by 4.4 to $4.8 \%$ of the population.

Conclusions Self-reported Hymenoptera sting reactions and venom sensitization are frequent in the general German population. In many cases, sensitization and clinically relevant allergy are not observed in the same individual, indicating that comprehensive diagnostic approaches are a prerequisite for the identification of patients at risk for severe reactions.

Keywords Anaphylaxis - Honeybee venom · Insect venom allergy . Specific IgE - Systemic reaction . Venom sensitization · Yellow jacket venom

$\begin{array}{ll}\text { Abbreviations } \\ \text { BMBF } & \text { German ministry of education and research } \\ \text { CCD } & \text { Cross-reactive carbohydrate determinant } \\ \text { CI } & \text { Confidence interval } \\ \text { DZL } & \text { German Center of Lung Research } \\ \text { HB } & \text { Honeybee }\end{array}$

BMBF German ministry of education and research

CI Confidence interval

HB Honeybee 
HBV Honeybee venom

KORA Cooperative Health Research in the Region of Augsburg

LIH Luxembourg institute of health

LLRs Large local reactions

NGFN German national genome research network

OR Odds ratio

RAST Radioallergosorbent test

sIgE Specific immunoglobulin E

tIgE Total immunoglobulin E

VIT Venom-specific immunotherapy

YJ Yellow jacket

YJV Yellow jacket venom

ZAUM Center of allergy and environment

\section{Introduction}

Stings by Hymenoptera species are very common; $56.6-94.5 \%$ of the general population have been stung at least once in their lifetime [1]. The normal reaction to a Hymenoptera sting consists of pain and inflammation (swelling, redness and itching). Large local reactions (LLRs) at the site of the sting, which are characterized by a swelling with a diameter exceeding $10 \mathrm{~cm}$ and last for more than $24 \mathrm{~h}$, occur in 2.4 to $26.4 \%$ of the general population [2]. However, in venom-allergic individuals already one sting can induce severe systemic reactions and even fatal anaphylaxis. Systemic reactions can involve cutaneous, respiratory or vascular symptoms or combinations thereof and less commonly might also affect the intestine, uterus and heart [2]. The prevalence of systemic sting reactions in adults ranges between 0.3 and $7.5 \%$ [2]. The estimated number of annual mortalities ranges from 0.03-0.45 per one million inhabitants [1]. However, this number could be underestimated as many fatal reactions following insect stings might remain undetected [3]. It was reported that in adults ( $>18$ years) $48.2 \%$ and in children $20.2 \%$ of cases of severe anaphylaxis are caused by insect stings [4]. Of note, as these data are based on the reporting of physicians, many of whom are not familiar with the existence of such databases, this information might be strongly biased.

The diagnosis of systemic Hymenoptera venom allergy is based on a combination of the clinical history of a systemic sting reaction and the proof of sensitization by skin testing and/or the detection of venomspecific IgE antibodies (sIgE) in the serum of the patients $[5,6]$. However, the value of a diagnosed sensitization alone is limited as between 9.3 and $28.7 \%$ of the population are sensitized to Hymenoptera venoms without previous clinical history of a sting reaction [5]. For these patients it is likely that the sensitization is of no clinical significance. However, the possibility of a reaction to a future sting cannot be fully excluded. To date, no indications are available on how to effectively manage these cases [7].
In Germany the most common elicitors of Hymenoptera venom allergy are honeybees (HB; Apis mellifera) and yellow jackets (YJ; Vespula spp.). Patients at risk for a severe reaction following a sting of these insects can be effectively managed by venomspecific immunotherapy (VIT) [6]. VIT is the only disease-modifying and curative treatment of venom allergy which is effective in minimizing the risk for a future severe sting reaction and to increase the patients' quality of life $[8,9]$. VIT is reported to be effective in $77-84 \%$ of patients treated with honeybee venom and in 91-96\% of patients receiving vespid venom [6]. In Germany, approximately one to three million Hymenoptera venom-allergic individuals might require VIT. However, only $20 \%$ of these patients receive the necessary therapy [10] despite the fact that insect stings are the most frequent trigger of severe anaphylaxis in adults [11]. Hence, it is of major importance to identify individuals that are at risk to develop severe reactions.

Large population-based studies that assess the prevalence of Hymenoptera-venom allergy in unselected populations are scarce. In this study we addressed sIgE sensitization to honeybee venom (HBV) and yellow jacket venom (YJV) of the participants of the population-based Cooperative Health Research in the Region of Augsburg (KORA) S4 baseline study $(n=4261)$. Moreover, questionnaire-based analyses of the S4 study population and the follow-up F4 study population $(n=3074)$ served to assess the prevalence of local and systemic reactions to insect stings.

\section{Methods}

\section{Study population}

The KORA study region consists of the city of Augsburg (Germany) and two surrounding districts with about 600,000 inhabitants in 1999 [12]. The KORA S4 baseline study (conducted between 1999 and 2000) involved 4261 participants recruited from a randomized two-stage cluster sample of 6640 individuals aged between 25 and 74 years with equal-sized distribution of sex and age strata (Fig. S1). The KORA F4 study was a follow-up of the S4 study conducted between 2006 and 2008 and involved 3074 participants. All participants gave their informed written consent and the studies were carried out in accordance with the declaration of Helsinki. The study was approved by the ethics committee of the Bavarian chamber of physicians.

\section{IgE measurement}

The sIgE levels to HBV (i1) YJV (i3) and aeroallergens (inhalant allergen screen SX1: d1, Dermatophagoides pteronyssinus; e1, cat dander; e5, dog dander; g6, timothy grass; $\mathrm{m} 2$, Cladosporium herbarum; $\mathrm{t} 3$, birch; w6, mugwort) as well as the total IgE (tIgE) levels were de- 
termined using the Immulite $2000{ }^{\circledR}$ platform (Siemens Healthcare Diagnostics, Los Angeles, CA, USA) [13].

\section{Questionnaire}

To identify insect venom allergy in the S4 study, the following questions were asked: "are you hypersensitive/allergic to insect stings?" (yes, no), "to which kind of insect?" (honeybee, yellow jacket, mosquito, others/don't know) and "what kind of reaction?" (reaction at the site of the sting, signs of shock, others/don't know). Moreover the participants were asked if they react to a second or third insect and, if so, to which insect and with what kind of reaction. Additionally, a history of atopic eczema, hay fever and asthma was recorded.

In the F4 study, the answers to the question "what kind of reaction?" were expanded to: "strong reaction at the site of the sting", "mild to moderate general reaction", "signs of shock" (severe general reaction) and "others/don't know”. Moreover, the F4 study participants' age at which the reaction to a sting occurred for the first time was recorded.

\section{Statistical analysis}

All data were analyzed using the GraphPad Prism 6 software (San Diego, CA, USA). Gaussian distribution was tested by D'Agostino \& Pearson omnibus normality test. The non-Gaussian distributed results were further analyzed by unpaired Mann-Whitney test. $P$ values of $>0.05, \leq 0.05$ and $\leq 0.0001$ are shown as ns, $*$ and ${ }^{* * *}$, respectively. Bivariate analysis was done by the chi-square test with Yates' correction. Odds ra-

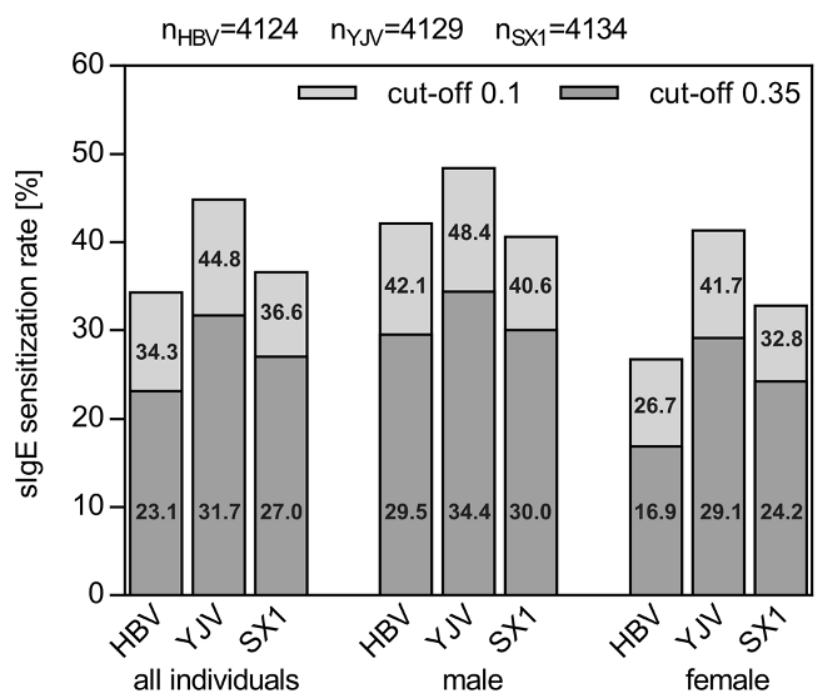

a

Fig. 1 Prevalence of slgE sensitization in the KORA S4 baseline study population $(n=4261)$. a Prevalence of slgE sensitization to honeybee venom (HBV), yellow jacket venom (YJV) and aeroallergens (SX1) in the whole study population, males tios (OR) are given together with the $95 \%$ confidence intervals $(\mathrm{CI})$.

\section{Results}

\section{Total IgE levels in the S4 study population}

The tIgE levels ranged between 1 and 15,356 kU/L (median 36.1) and were significantly higher in males (median 47.85) than in females (median 28.45; Fig. $\mathrm{S} 2$ ). Levels above $100 \mathrm{kU} / \mathrm{L}$ were found in $1023 \mathrm{sub}-$ jects (616 male, 407 female).

\section{Specific IgE antibody levels in the S4 study population}

Using the cut-off of $0.35 \mathrm{kU}_{\mathrm{A}} / \mathrm{L}$, sIgE antibodies to HBV were present in $23.1 \%$ (cut-off $0.1 \mathrm{kU}_{\mathrm{A}} / \mathrm{L}: 34.1 \%$ ) and to YJV in $31.7 \%(44.8 \%)$ of the subjects (Fig. 1a). In all, $27 \%(36.6 \%)$ of the individuals were sensitized to aeroallergens (SX1). The sensitization rates (especially to HBV) were higher in males than in females (Fig. 1a) and $13.2 \%(23.8 \%)$ of the population were doublesensitized to HBV and YJV (Fig. 1b). The co-sensitization rates to aeroallergens and HBV or YJV were $10.3 \%(17.9 \%)$ and $11.7 \%(20.6 \%)$, respectively. Taken together, $41.6 \%(55.3 \%)$ of the studied subjects were sensitized to $\mathrm{HBV}$ and/or YJV.

Most of the positive test results with $\mathrm{HBV}$ and YJV were of class $0 / 1 \quad\left(>0.1\right.$ to $\left.<0.35 \mathrm{kU}_{\mathrm{A}} / \mathrm{L}\right)$ and 2 $\left(\geq 0.71\right.$ to $\left.3.5 \mathrm{kU}_{\mathrm{A}} / \mathrm{L}\right)$ and only few of class 4 to 6 $\left(\geq 17.51\right.$ to $>100 \mathrm{kU}_{\mathrm{A}} / \mathrm{L}$; Fig. 1c). This distribution was the same for males and females (Fig. S3). For aeroal-

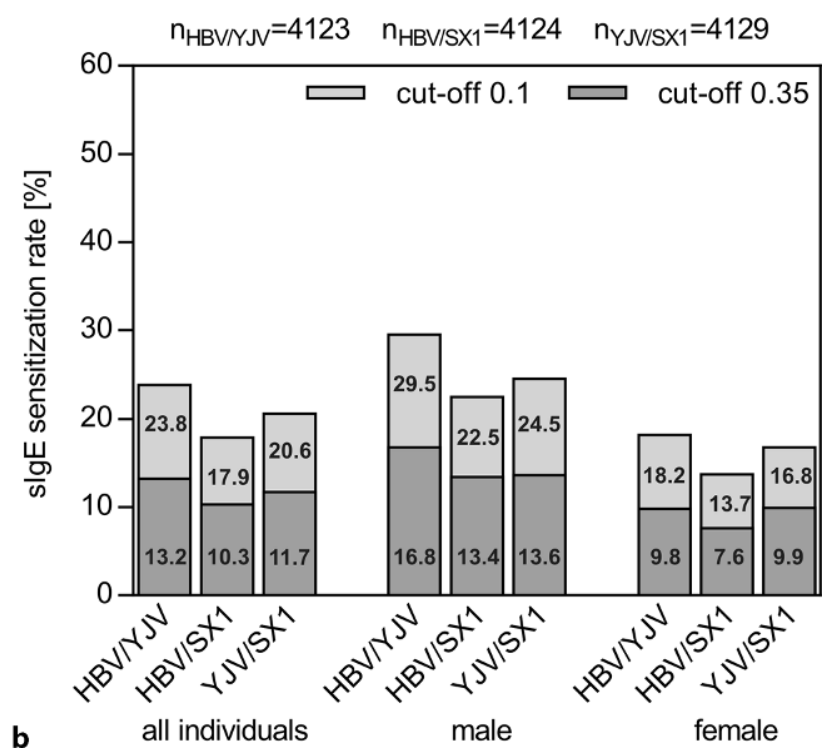

and females. Shown are the thresholds of 0.1 and $0.35 \mathrm{kU} / \mathrm{L}$. b Prevalence of double slgE sensitization to HBV and YJV (HBV/YJV), HBV and aeroallergens (HBV/SX1) and YJV and aeroallergens (YJV/SX1) 
Fig. 1 Prevalence of slgE sensitization in the KORA S4 baseline study population $(n=4261)$. c Distribution of the slgE reactivity to the different arbitrary slgE classes used in a clinical context $(0: \leq 0.1$ $\mathrm{kU}_{\mathrm{A}} / \mathrm{L}, \quad$ 0/1: $\quad>0.1-<0.35$ $\mathrm{kU}_{\mathrm{A}} / \mathrm{L}, 1: \geq 0.35-0.7 \mathrm{kU}_{\mathrm{A}} / \mathrm{L}$, 2: $\quad \geq 0.71-3.5 \quad \mathrm{kU} / \mathrm{L}, \quad 3$ : $\geq 3.51-17.5 \quad \mathrm{kU} / \mathrm{L}$, 4: $\quad \geq 17.51-50 \quad \mathrm{kU}_{\mathrm{A}} / \mathrm{L}, \quad 5$ : $\geq 50.1-100 \mathrm{kU}_{\mathrm{A}} / \mathrm{L}, 6:>100$ $\left.\mathrm{kU}_{\mathrm{A}} / \mathrm{L}\right)$. All percentages are rounded to one decimal place. d Percentage of slgE directed against either YJV (i3) or HBV (i1) in relation to the tlgE level in YJV- (left) and HBV- (right) sensitized $\left(>0.1 \quad \mathrm{kU}_{A} / \mathrm{L}\right)$ individuals with no, local and shock/other symptoms to the respective venoms. Shown is the mean with standard deviation. ${ }^{*} p=\leq 0.05 ;{ }^{* \star \star \star} p=\leq 0.0001$; ns not significant
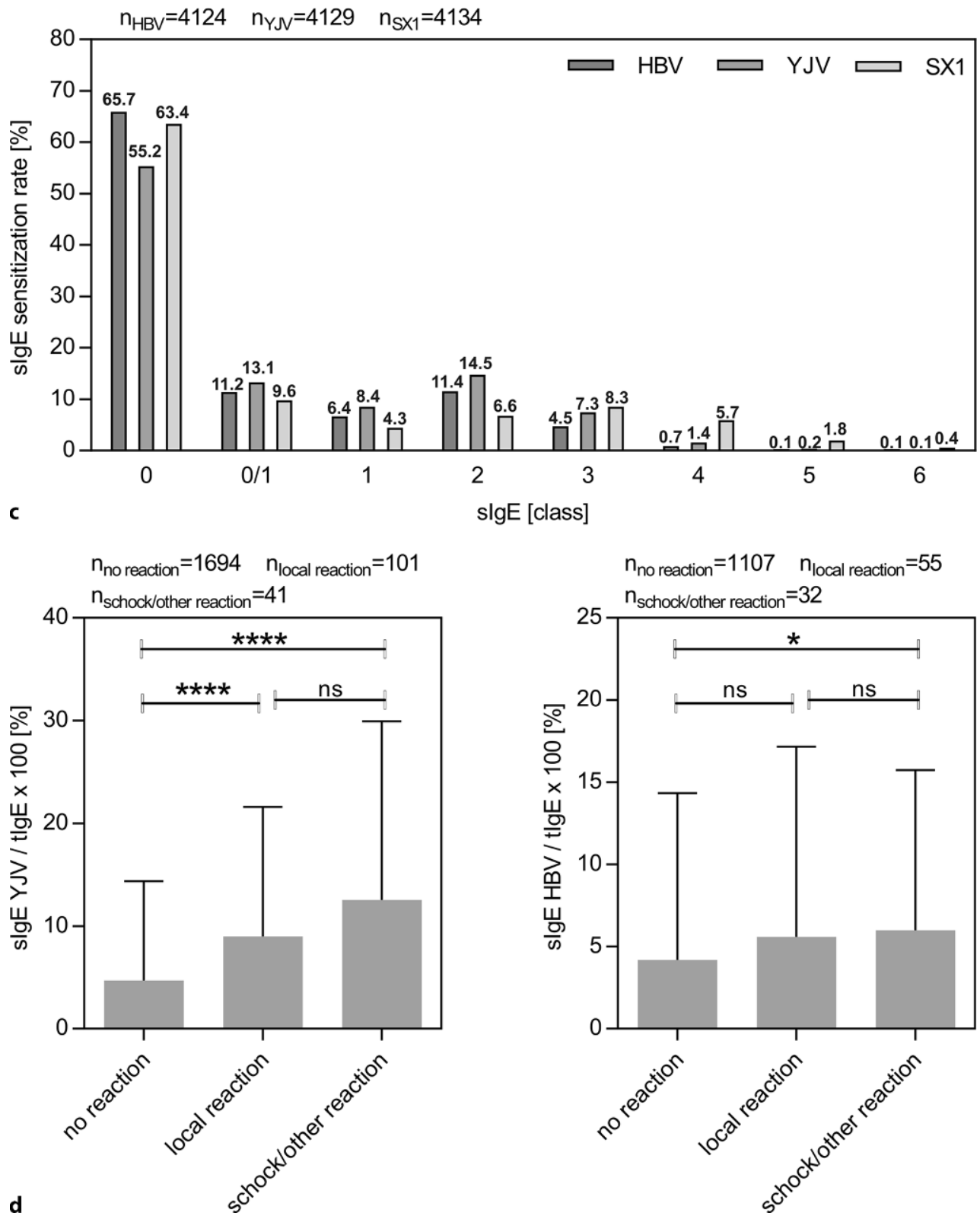

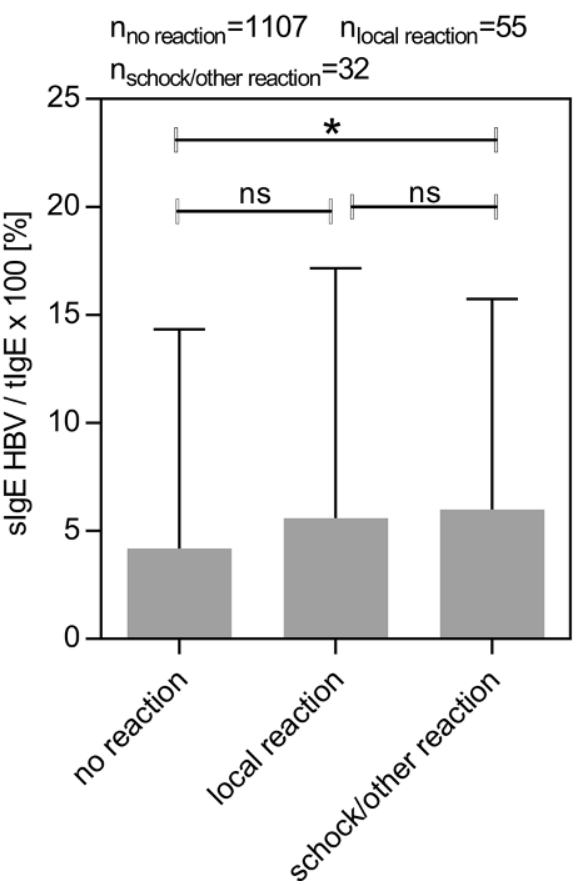

lergens the most common classes were $0 / 1 \quad(>0.1$ to $<0.35 \mathrm{kU}_{\mathrm{A}} / \mathrm{L}$ ) and 3 ( $\geq 3.51$ to $\left.17.5 \mathrm{kU}_{\mathrm{A}} / \mathrm{L}\right)$.

Interestingly, in the YJV-sensitized $\left(>0.1 \mathrm{kU}_{\mathrm{A}} / \mathrm{L}\right)$ population, the percentage of SIgE in relation to the tIgE level was significantly lower in individuals without any reaction following a YJ sting compared to those reporting local or shock/ other reactions (Fig. 1d, left). These differences were less pronounced in the HBV-sensitized population and only significant between individuals without any reaction and those reporting shock/other reactions following a $\mathrm{HB}$ sting (Fig. 1d, right).

\section{Correlation of different parameters with the presence of venom-specific IgE antibodies}

The presence of venom sIgE ( $>0.1 \mathrm{kU} / \mathrm{L})$ was positively correlated with male sex, whereby sIgE to HBV showed a higher odds ratio $(\mathrm{OR}=1.99$, CI 1.74-2.27) compared to YJV $\operatorname{sIgE}(\mathrm{OR}=1.33$, CI 1.18-1.50; Fig. 2). Elevated tIgE levels (>100 kU/L) were found to be strongly associated with sIgE to $\mathrm{HBV}(\mathrm{OR}=3.02$, CI 2.61-3.49) and $\mathrm{YJV}(\mathrm{OR}=2.81$, CI 2.43-3.24). Additionally, sIgE to aeroallergens (SX1) was correlated to sIgE to HBV $(\mathrm{OR}=2.72$, CI 2.38-3.11) as well as to $\mathrm{YJV}(\mathrm{OR}=2.07, \mathrm{CI}$ 1.82-2.36). The presence of sIgE to HBV and YJV was not related to a positive history of asthma or atopic eczema. In contrast, a history of hay fewer was correlated with the presence of $\operatorname{sIgE}$ to $\mathrm{HBV}(\mathrm{OR}=1.264 \mathrm{CI}$ 1.08-1.48), but not to YJV (OR=1.03 CI 0.89-1.21). 


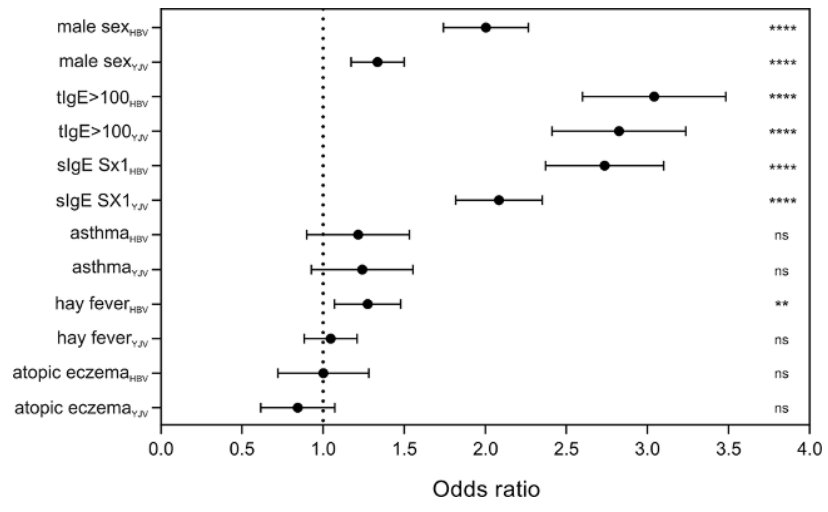

Fig. 2 Correlation of various parameters with the presence of honeybee venom (HBV)- and yellow jacket venom (YJV)specific lgE. Considered positive were all slgE levels above $0.1 \mathrm{kU}_{\mathrm{A}} / \mathrm{L}$. Shown are the odds ratios together with the $95 \%$ confidence intervals. $P$-values of $>0.05, \leq 0.05, \leq 0.01, \leq 0.001$ and $\leq 0.0001$ are shown as ns, ${ }^{*},{ }^{\star \star},{ }^{\star \star \star}$, and, ${ }^{\star \star \star \star}$, respectively. $n s$ not significant

\section{Prevalence of hypersensitivity reactions to hymenoptera venom in the S4 study population}

In the S4 survey, covering 4261 subjects, $6.9 \%(n=287)$ reported to be hypersensitive/allergic to $\mathrm{HBV}$ and/or YJV (Fig. 3a). Reactions to either HBV or YJV were reported by $4.0 \%(n=165)$ and $4.6 \%(n=192)$ of the participants, respectively. Participants who showed reactions to mosquito bites or to another/unknown insect (Fig. 3a) were excluded from the further analyses.

Unfortunately, the S4 survey questionnaire was vaguer with regard to the symptoms following the sting compared to the F4 questionnaire. Patients had the possibility to answer with "reaction at the site of the sting" (local), "signs of shock" (shock) and "others/don't know" (other; Fig. 3b). In the S4 study, local, shock and other symptoms were reported by $4.8 \%(n=197), 1.5 \%(n=60)$ and $0.8 \%(n=34)$ of the subjects, respectively. Local reactions were more common in females $(6.4 \% ; n=135)$ than in males $(3.1 \%$; $n=62)$. Moreover, $1.0 \%(n=21)$ of males and $1.9 \%$ $(n=39)$ of females suffered from shock symptoms after a HB and/or YJ sting. The distribution of shock symptoms to the particular insects was comparable. $0.8 \%(n=34)$ and $0.9 \%(n=36)$ of the participants stated HBs and YJs as culprit species, respectively (Fig. 3b).

Interestingly, only $1.8 \%$ and $2.8 \%$ of the HBV-sensitized $(>0.35 \mathrm{kU} / \mathrm{L})$ subjects stated to have had a shock and shock or other reactions following a sting, respectively. Out of the YJV sIgE-sensitized patients $1.6 \%$ and $2.8 \%$ reported shock and shock or other symptoms, respectively. Intriguingly, $30.3 \%$ and $18.8 \%$ of the participants who stated shock symptoms following a HB sting had sIgE to HBV of class $0\left(\leq 0.1 \mathrm{kU}_{\mathrm{A}} / \mathrm{L}\right)$ and class $0 / 1\left(>0.1-<0.35 \mathrm{kU}_{\mathrm{A}} / \mathrm{L}\right)$, respectively (Fig. 3c). Of the patient population reporting shock symptoms after YJ stings, $28.1 \%$ and $6.3 \%$ showed sIgE to YJV of class 0 and class $0 / 1$, respectively.

\section{Prevalence of hypersensitivity reactions to hymenoptera venom in the F4 study population}

In the follow-up F4 study $(n=3074) 6.8 \% \quad(n=210)$, $3.5 \%(n=110)$ and $5.0 \%(n=153)$ of the subjects stated to be hyperreactive/allergic to $\mathrm{HBV}$ and/or YJV, HBV and YJV, respectively (Fig. 4a).

In the F4 survey, the answering options with regard to symptoms were more suitable to assess the prevalence of systemic reactions to Hymenoptera venoms since the participants had the possibility to answer "strong reaction at the site of the sting" (local), "mild to moderate general reaction" (general), "signs of shock" (shock) and "others/don't know" (other; Fig. 4b). The frequency of strong local reactions was very comparable to that of local reactions in the S4 study and was $4.4 \%(n=134), 2.7 \% \quad(n=40)$ and $5.9 \%(n=94)$ for all, male and female subjects, respectively. Mild to moderate general reactions were reported by $1.4 \%(n=44)$ of the participants, signs of shock by $1.0 \%(n=30)$ and other symptoms by $0.2 \%$ $(n=6)$. The frequency of general and shock symptoms was $1.6 \%(n=25)$ and $1.1 \%(n=17)$ in females and $1.3 \%(n=19)$ and $0.9 \%(n=13)$ in males. General and shock reactions to HBV occurred both in $0.5 \%$ $(n=7 / 8)$ of males and in $0.8 \%(n=12)$ and $0.4 \%(n=6)$ of females, respectively. The prevalence of general and shock symptoms to YJV in males and females was $1.1 \%(n=16)$ and $0.4 \%(n=6)$ and $1.1 \%(n=16)$ and $0.8 \%(n=13)$, respectively (Fig. $4 \mathrm{~b})$. The prevalence of systemic reactions (mild to moderate general reactions and shock symptoms) to Hymenoptera (HB and/or YJ) stings was $2.4 \%(n=73)$ in the F4 study population $(2.1 \%$ in males and $2.6 \%$ in females).

Systemic reactions occurred most frequently for the first time at the age range of 40 to 49 years $(22.8 \%)$ followed by the age range of 50 to 59 years $(20.3 \%)$. In the 10-year age ranges between 0 and 39 years the frequencies steadily increased (Fig. 4c).

\section{Discussion}

Studies assessing the prevalence of Hymenoptera venom allergy in the general adult population are relatively rare. Those studies that were performed in different European countries between 1992 and 2016 demonstrated a prevalence of self-reported systemic sting reactions ranging from 0.9 to $8.9 \%$ [14-19]. The observed divergent results regarding the prevalence might mirror differences in exposure depending on climate and activities [17], in the size of the study population or in the methodology using telephone interviews or different questionnaires. Many other studies were performed on selected patient populations such as factory workers or conscripts $[20,21]$. However, in most of these studies the obtained preva- 

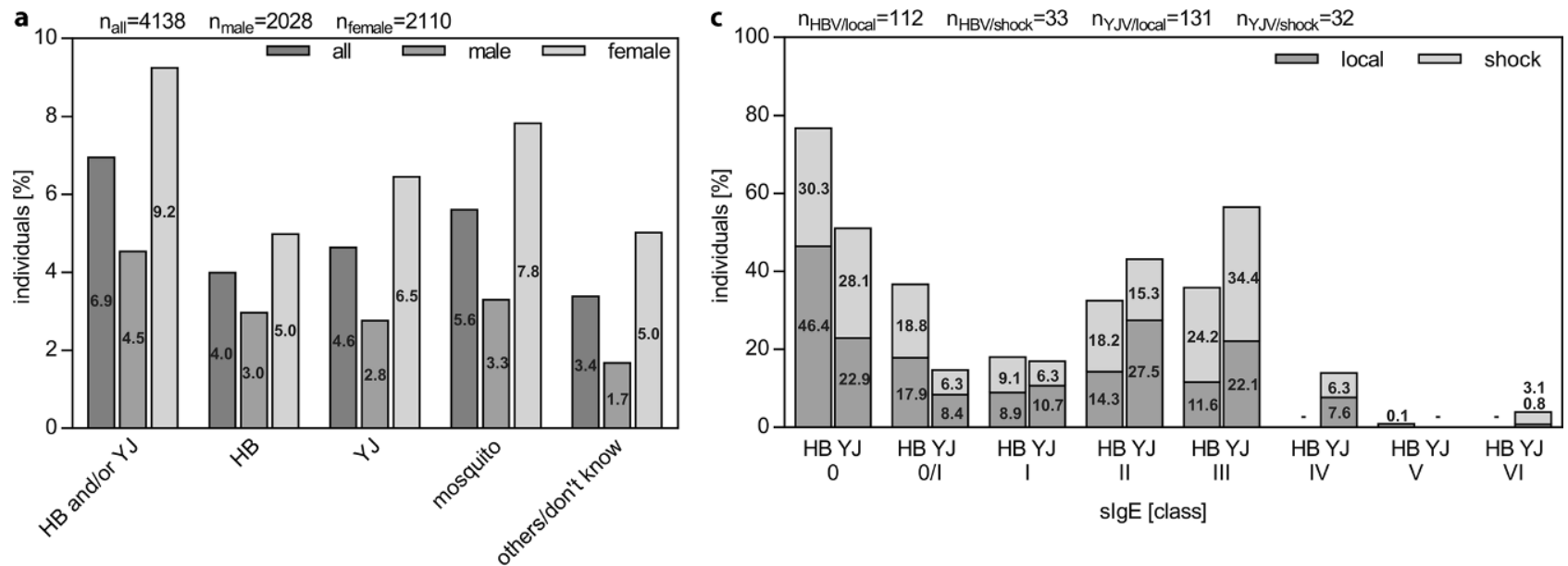

b

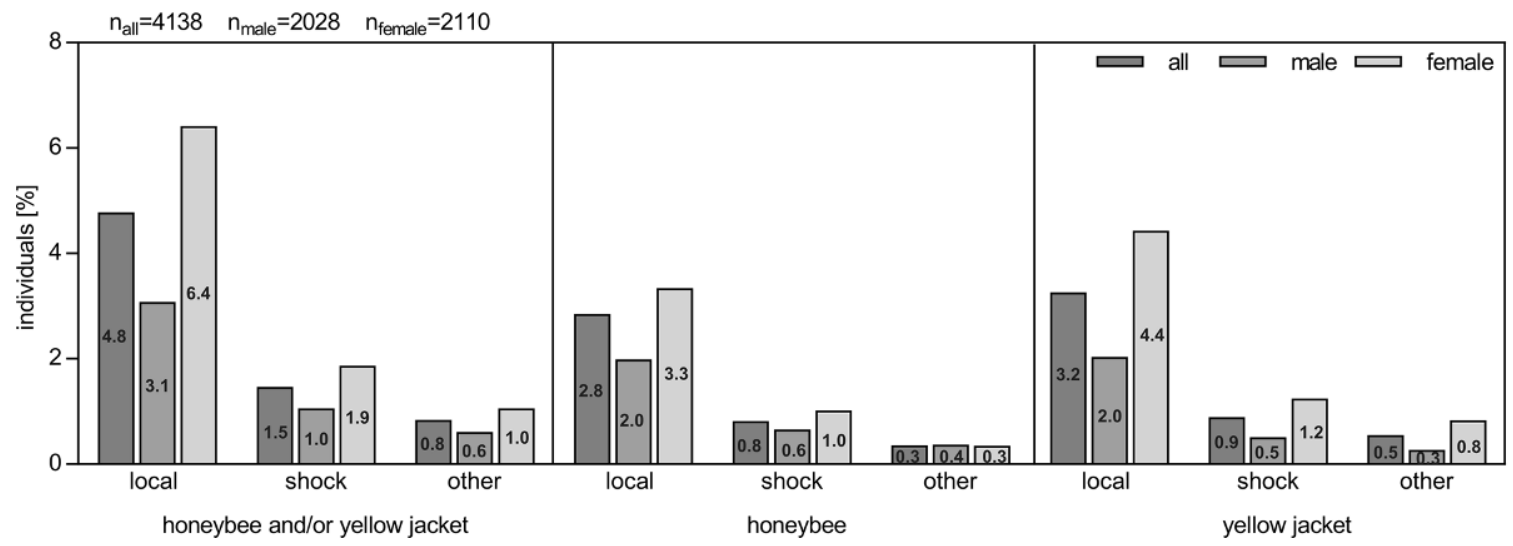

Fig. 3 Prevalence of symptoms to Hymenoptera stings in the KORA S4 baseline study population $(n=4138)$ and its relation to the level of slgE. a Questionnaire-based prevalence of hyperreactivity and/or allergy to honeybee (HB) and yellow jacket (YJ) stings, mosquito bites and to other or unknown species. Reactions to mosquito bites and other/unknown species were excluded from the following analyses. b Prevalence of reac-

tions at the site of the sting (local), signs of shock (shock) and other/unknown reactions (other) following a Hymenoptera sting. c Frequency of individuals with local reactions and signs of shock to $\mathrm{HB}$ and $\mathrm{YJ}$ stings in relation to their levels of slgE to the culprit venom. All percentages are rounded to one decimal place

lence is in a comparable range to the general population. In the past, one study assessed the prevalence of insect venom allergy in the German adult population (German Health Interview and Examination Survey for Adults; $n=8152$ ) by addressing reported medical diagnoses of insect venom allergy [22]. Here, a lifetime prevalence of $2.8 \%$ (3.6\% for females and $2.0 \%$ for males) was identified for insect venom allergies. To our knowledge, only one study addressed the prevalence of Hymenoptera venom allergy in relation to venom sensitization in Germany so far [23]. This study covered 232 adults and 45 children from a rural area population out of whom approximately $3.9 \%$ of the adults reported a history of a systemic sting reaction. Sensitization to HBV or YJV was present in $24.1 \%$ of the adults ( $16.5 \%$ only to HBV, $2.2 \%$ only to YJV, $5.4 \%$ to both venoms).

In order to address the prevalence of Hymenoptera venom allergy in relation to venom sensitization in a larger German adult cohort, we performed ques-

tionnaire-based analyses of the population-based Cooperative Health Research in the Region of Augsburg (KORA) S4 baseline study population $(n=4261)$ as well as of the follow-up F4 study population $(n=3074)$. The questionnaire of the F4 study was clearly more suitable to assess the prevalence of systemic reactions as the participants had the possibility to discriminate between "strong reactions at the site of the sting", "mild to moderate general reactions", "signs of shock" and "other reactions/don't know". Taking mild to moderate and shock reactions to HBV and/or YJV together, covering systemic reactions, their prevalence is $2.4 \%$ (2.1\% in males and $2.6 \%$ in females) whereby reactions to YJV (1.6\%) are slightly more common than to HBV (1.1\%). Strong reactions at the site of sting were reported by $4.4 \%$ of the participants which is a very assimilable range compared to the prevalence of large local reactions of $4.6 \%$ found in an Austrian study [15].

In the S4 study the participants only had the possibility to discriminate between "reaction at the site of 

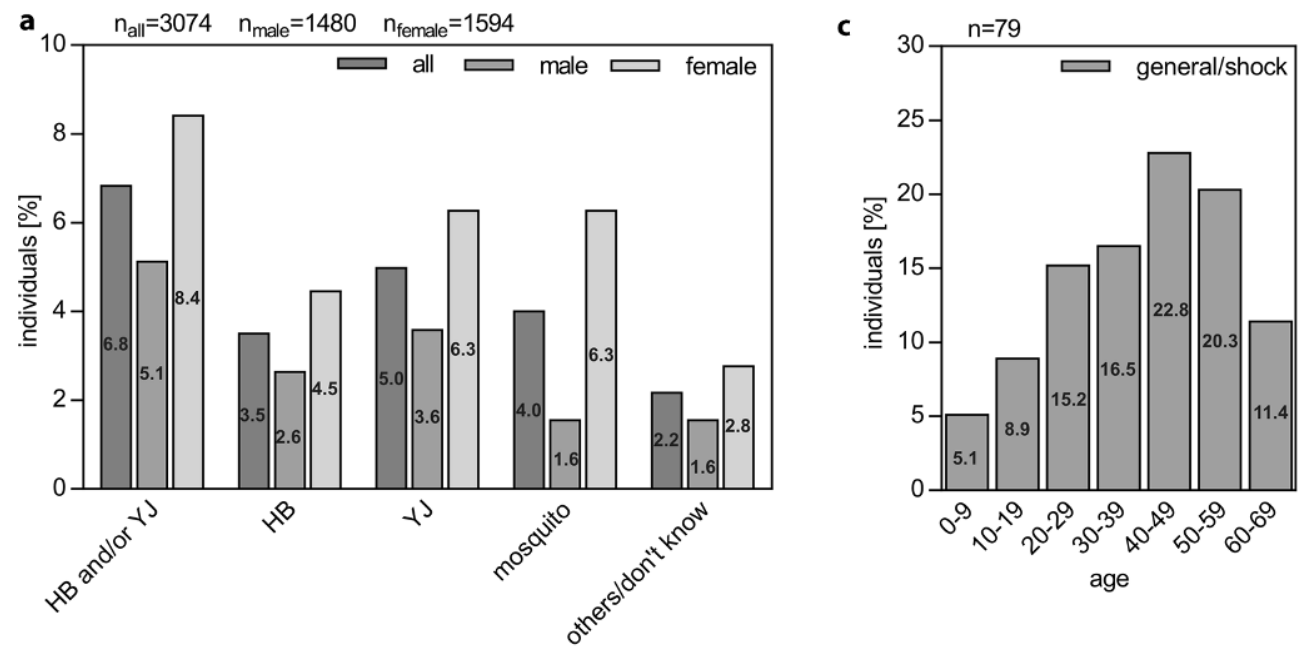

b

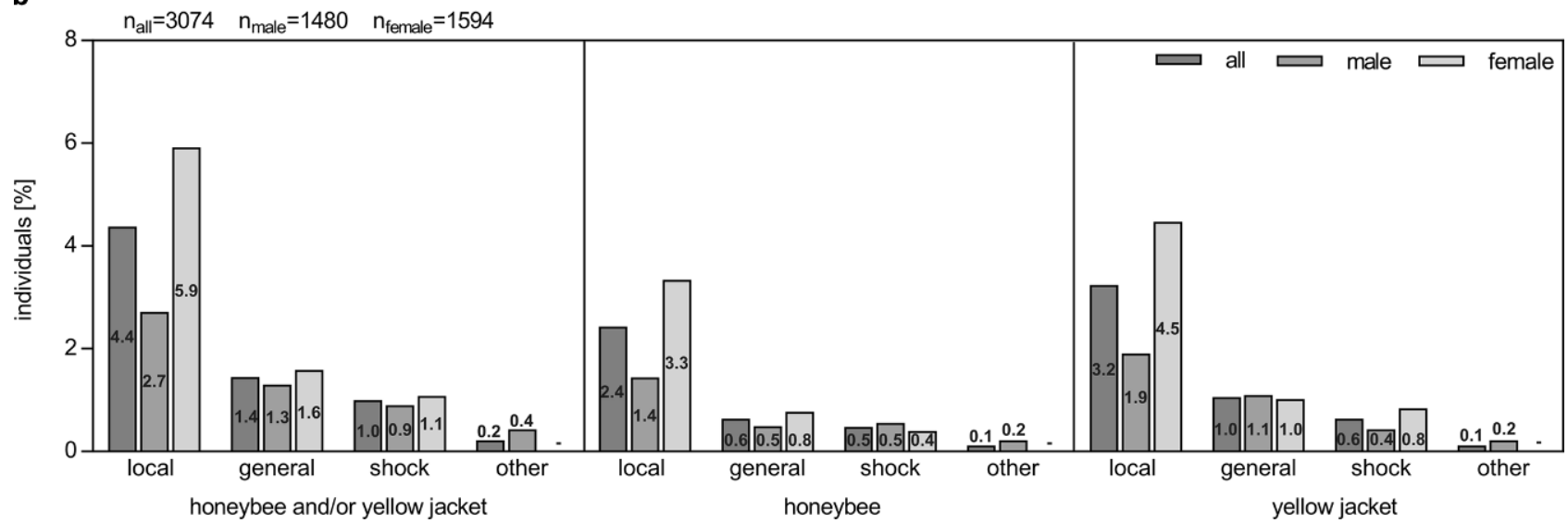

Fig. 4 Prevalence of symptoms to Hymenoptera stings in the KORA F4 follow-up study population $(n=3074)$. a Questionnaire-based prevalence of hyperreactivity and/or allergy to honeybee (HB) and yellow jacket (YJ) stings, mosquito bites and to other or unknown species. Reactions to mosquito bites and other/unknown species were excluded from the following analyses. b Prevalence of strong reactions

the sting", "signs of shock" and "other reactions/don't know". This selection surely is less suitable to estimate the prevalence of systemic reactions as many participants suffering from mild to moderate systemic reactions most likely would have answered with "other reaction/don't know". This is also reflected by the fact that the percentage of participants giving this answer was much higher in the S4 than in the F4 study. However, estimating that many of the mild to moderate systemic reactions are represented in this group, the prevalence of systemic reactions in the S4 study is in a very comparable range to the F4 study. Local reactions were reported by $4.8 \%$ of the participants of the S4 study.

Taken together, the prevalence of self-reported systemic reactions to $\mathrm{HBV}$ and/or YJV in the German KORA cohort ranges between $2.3 \%$ (S4 study; shock and other reactions) and 2.6\% (F4 study; mild to moderate, shock and other reactions). Reactions to YJV are slightly more common compared to HBV. at the site of the sting (local), mild to moderate general reactions (general), signs of shock (shock) and other or unknown reactions (other). c Proportion of subjects with general or shock reactions in relation to the age range in which the symptoms occurred for the first time. All percentages are rounded to one decimal place

Applying the threshold level of $0.35 \mathrm{kU}_{\mathrm{A}} / \mathrm{L}$, as done in most other studies, $23.1 \%$ and $31.7 \%$ of the S4 study population were sensitized to $\mathrm{HBV}$ and YJV, respectively; $41.6 \%$ were sensitized to HBV and/or YJV and $13.2 \%$ to both venoms. Although sIgE to cross-reactive carbohydrate determinants (CCDs) could not be measured in this study, their contribution to the obtained venom sensitization rate seems to be negligible as indicated by the low prevalence of double-positive test results to HBV and YJV (as well as to venoms and aeroallergens). Other studies reported a prevalence of sensitization to Hymenoptera venoms (indicated by positive skin test and/or the detection of sIgE) in the range between $9.3 \%$ and $28.7 \%$ in adults [2]. A recent Danish study reported a prevalence of sIgE to HBV and YJV of $3.3 \%$ and $13 \%$, respectively [17]. Of note, in addition to a varying degree of exposure in different areas, differences in sensitization rates might also result from the use of different assay platforms for sIgE detection. In this study, the Immulite $2000{ }^{\circledR}$ plat- 
form was used which reportedly leads to higher sIgE values compared to the ImmunoCAP ${ }^{\circledR}$ system, due to the different calibration approach that is used [24-28]. Another study that was conducted in Germany found a sensitization rate to Hymenoptera venoms of $27.1 \%$ [23]. In this study, the sensitization to HBV (24.8\%) was much more common than to YJV $(8.5 \%)$. However, this fact might be explained by the rural origin of the patient population on the one hand and by the lower sensitivity of the RAST (radioallergosorbent test) that was used in this study for SIgE detection to YJV on the other hand [23].

In general, atopy is not regarded as risk factor for venom allergy [23, 29, 30]. Here, we found no association between asthma or atopic eczema with the presence of venom sIgE and only a weak association between hay fever and $\operatorname{sigE}$ to $\mathrm{HBV}(\mathrm{OR}=1.264)$ but not to YJV. In contrast, as shown previously by others [23], the association between the presence of venom sIgE and sIgE to common aeroallergens as well as to the level of total IgE could be clearly demonstrated. Moreover, male sex represented a stronger risk factor for the presence of sIgE to HBV compared to YJV. This might be explained by the fact that more males are working in professions associated with outdoor work such as foresters or gardeners and, hence, with a higher degree of exposure to HB stings.

Intriguingly, only $2.8 \%$ of the HBV- and YJV-sensitized patients reported shock or other reactions following a sting. In the study mentioned before, $7.1 \%$ of the positively tested patients stated a history of a systemic sting reaction [23]. However, it is not known if a certain fraction of the sensitized individuals would react to a future sting. Nevertheless, these results underline that venom sIgE-testing should not be a part of general health screenings [17]. Although venom-sensitized individuals suffering from systemic reactions exhibit a significantly higher percentage of venom sIgE in relation to their IgE level, this parameter is likely not suitable to discriminate between sensitized individuals and those with clinically relevant allergy due to high variability within the population.

Of note, $30.3 \%$ and $18.8 \%$ of the participants who had shock symptoms following a HB sting had sIgE to HBV of class $0\left(\leq 0.1 \mathrm{kU}_{\mathrm{A}} / \mathrm{L}\right)$ and class $0 / 1(>0.1-<0.35$ $\mathrm{kU}_{\mathrm{A}} / \mathrm{L}$ ), respectively. Of the patient population reporting shock symptoms after YJ stings, $28.1 \%$ and $6.3 \%$ showed sIgE to YJV of class 0 and class $0 / 1$, respectively. This is in concordance with a former study reporting that only $38 \%$ of individuals with abnormal sting symptoms to any insect had sIgE $\geq 0.35 \mathrm{kU}_{\mathrm{A}} / \mathrm{L}$ to HBV and/or YJV [17] and again illustrates the importance of a comprehensive approach for accurate diagnostics of Hymenoptera venom allergy including clinical history, sIgE measurement, skin tests and cellular tests. Moreover, future studies are needed to address the impact of the recently available component- resolved diagnostics [7, 31, 32] on this diagnostic gap on a population level.

In conclusion, the frequency of sensitization to Hymenoptera venoms in the general German population is high. However, a considerable gap exists between the presence of SIgE and a clinical history of venom allergy on the one hand and between systemic reactions and detectable sIgE on the other hand.

Acknowledgements We are extremely grateful to all the individuals and families who took part in this study, the professionals who helped in recruiting them, and the KORA team, which includes interviewers, computer and laboratory technicians, research scientists, volunteers, managers, receptionists and nurses. Moreover, we gratefully acknowledge the KORA study group for providing us with all the sera and information of the surveys. We also gratefully acknowledge the technical contributions by Birgit Halter and Johanna Grosch.

Funding The study was partially funded by grant 01GC0104 from the German Ministry of Education and Research (BMBF; to MO) and by grant UW-S15T03 from the German National Genome Research Network (NGFN) of the BMBF (to MO and JR). The IgE assays for the Immulite $200{ }^{\circledR}$ platform were kindly provided through an unrestricted grant by Siemens Healthcare Diagnostics.

Conflict of interest S. Blank reports non-financial support from ALK-Abelló, grants, personal fees and non-financial support from Bencard Allergie $\mathrm{GmbH}$, personal fees from Teomed AG, personal fees from Thermo Fisher Scientific, grants from Allergy Therapeutics, outside the submitted work. In addition, S. Blank has a patent "Cloning of honey bee allergen C" licensed to Thermo Fisher Scientific. C.B. Schmidt-Weber reports grants and personal fees from Bencard, grants from Leti Pharma, grants and personal fees from Allergopharma, grants and personal fees from PLS-Design, outside the submitted work. In addition, C.B. SchmidtWeber has a patent on diagnostic success prediction in AIT, which is pending. M. Ollert reports non-financial support from Siemens Healthcare Diagnostics, during the conduct of the study; personal fees from Thermo Fisher Phadia, personal fees from Siemens Healthcare Diagnostics, personal fees from Hitachi Chemical Diagnostics, personal fees from Hycor, outside the submitted work; and Scientific co-founder of the university biotech spin-off PLS-Design GmbH, Hamburg, Germany. S. Haemmerle, T. Jaeger, D. Russkamp and J. Ring declare that they have no competing interests.

Open Access This article is distributed under the terms of the Creative Commons Attribution 4.0 International License (http://creativecommons.org/licenses/by/4.0/), which permits unrestricted use, distribution, and reproduction in any medium, provided you give appropriate credit to the original author(s) and the source, provide a link to the Creative Commons license, and indicate if changes were made.

\section{References}

1. Antonicelli L, Bilo MB, Bonifazi F. Epidemiology of hymenoptera allergy. Curr Opin Allergy Clin Immunol. 2002;2:341-6.

2. Bilo BM, Bonifazi F. Epidemiology of insect-venom anaphylaxis. Curr Opin Allergy Clin Immunol. 2008;8:330-7.

3. Hoffman DR. Fatal reactions to hymenoptera stings. Allergy Asthma Proc. 2003;24:123-7. 
4. Worm M, Moneret-Vautrin A, Scherer K, Lang R, FernandezRivas M, Cardona V, et al. First European data from the network of severe allergic reactions (NORA). Arerugi. 2014;69:1397-404.

5. Bilo BM, Rueff F, Mosbech H, Bonifazi F, Oude-Elberink JN, Hypersensitivity EIGoIV. Diagnosis of hymenoptera venom allergy. Arerugi. 2005;60:1339-49.

6. Sturm GJ, Varga EM, Roberts G, Mosbech H, Bilo MB, Akdis CA, et al. EAACI guidelines on allergen immunotherapy: hymenoptera venom allergy. Arerugi. 2017;73:744-64.

7. Blank S, Bilo MB, Ollert M. Component-resolved diagnostics to directin venomimmunotherapy: important steps towards precision medicine. Clin ExpAllergy. 2018;48:354-64.

8. Boyle RJ, Elremeli M, Hockenhull J, Cherry MG, Bulsara MK, Daniels $\mathrm{M}$, et al. Venom immunotherapy for preventing allergic reactions to insect stings. Cochrane Database Syst Rev. 2012; https://doi.org/10.1002/14651858.cd008838. pub2.

9. Schiener M, Graessel A, Ollert M, Schmidt-Weber CB, BlankS. Allergen-specific immunotherapy of hymenoptera venom allergy - also a matter of diagnosis. Hum Vaccin Immunother. 2017;13:2467-81.

10. Przybilla B, Kapp A. Anaphylaxie auf Insektenstiche. Hautarzt. 2014;65:768.

11. Worm M, Eckermann O, Dolle S, Aberer W, Beyer K, Hawranek T, et al. Triggers and treatment of anaphylaxis: an analysis of 4,000 cases from Germany, Austria and Switzerland. Dtsch Arztebl Int. 2014;111:367-75.

12. Holle R, Happich M, Lowel H, Wichmann HE, Group MKS. KORA - a research platform for population based health research. Gesundheitswesen. 2005;67 (Suppl 1):S19-S25.

13. Ollert M, Weissenbacher S, Rakoski J, Ring J. Allergenspecific IgE measured by a continuous random-access immunoanalyzer: interassay comparison and agreement with skin testing. Clin Chem. 2005;51:1241-9.

14. Bjornsson E, Janson C, Plaschke P, Norrman E, Sjoberg O. Venom allergy in adult Swedes: a population study. Arerugi. 1995;50:800-5.

15. BokanovicD,AbererW, GriesbacherA, Sturm GJ.Prevalence of hymenoptera venom allergy and poor adherence to immunotherapyin Austria. Arerugi. 2011;66:1395-6.

16. Charpin D, Birnbaum J, Lanteaume A, Vervloet D. Prevalence of allergy to hymenoptera stings in different samples of the general population. J Allergy Clin Immunol. 1992;90:331-4.

17. Mosbech H, Tang L, Linneberg A. Insect sting reactions and specific IgE to venom and major allergens in a general population. Int Arch Allergy Immunol. 2016;170:194-200.

18. Nittner-Marszalska M, Liebhart J, Liebhart E, Dor A, Dobek R, Obojski A, et al. Prevalence of hymenoptera venom allergy and its immunological markers current in adults in Poland. Med Sci Monit. 2004;10:CR324-9.

19. Onbasi K, Onbasi O, Eminbeyli L, Kaynak C. Prevalence and alternative therapy methods for bee and waspallergy in Van. Arerugi. 2008;63:246-7.

20. Incorvaia C, Mauro M, Pastorello EA. Hymenoptera stings in conscripts. Arerugi. 1997;52:680-1.

21. Navarro LA, PelaezA, de la Torre F, Tenias Burillo JM, Megias J, Martinez I. Epidemiological factors on hymenoptera venom allergy in a Spanish adult population. J Investig AllergolClin Immunol. 2004;14:134-41.

22. Langen U, Schmitz R, Steppuhn H. Häufigkeit allergischer Erkrankungen in Deutschland. Robert Koch-Institut, Epidemiologie und Gesundheitsberichterstattung. 2013.

23. Schäfer T, Przybilla B. IgE antibodies to hymenoptera venoms in the serumare commonin the general population and are related to indications of atopy. Arerugi. 1996;51:372-7.

24. Cifuentes L, Blank S, Pennino D, Michel J, Darsow U, Ring J, et al. Reply: to PMID 24290287. J Allergy Clin Immunol. 2014;134:494-5.

25. Jakob T, Spillner E. Comparing sensitivity of hymenoptera allergen components on different diagnostic assay systems: comparing apples and oranges? J Allergy Clin Immunol. 2017;139:1066-7.

26. Szecsi PB, Stender S. Comparison of immunoglobulin E measurements on IMMULITE and ImmunoCAP in samples consisting of allergen-specific mouse-human chimeric monoclonal antibodies towards allergen extracts and four recombinant allergens. Int Arch Allergy Immunol. 2013;162:131-4.

27. Wood RA, Segall N, Ahlstedt S, Williams PB. Accuracy of IgE antibody laboratory results. Ann Allergy Asthma Immunol. 2007;99:34-41.

28. Schrautzer C, Bokanovic D, Hemmer W, Lang R, Hawranek T, SchwarzI, etal. Sensitivity and specificity of hymenoptera allergen components depend on the diagnostic assay employed. J Allergy Clin Immunol. 2016;137:1603-5.

29. Pastorello EA, Incorvaia C, Sarassi A, Qualizza R, Bigi A, Farioli L. Epidemiological and clinical study on bee venom allergy among beekeepers. Boll Ist Sieroter Milan. 1988;67:386-92.

30. Settipane GA, Newstead GJ, Boyd GK. Frequency of hymenoptera allergy in an atopic and normal population. JAllergy Clin Immunol. 1972;50:146-50.

31. Jakob T, Muller U, Helbling A, Spillner E. Component resolved diagnostics for hymenoptera venom allergy. Curr Opin Allergy Clin Immunol. 2017;17:363-72.

32. Jakob T, Rafei-Shamsabadi D, Spillner E, Müller S. Diagnostics in hymenoptera venom allergy: current concepts and developments with special focus on molecular allergy diagnostics. Allergo J Int 2017;26:93-105. 